\title{
Verfahren wirtschaftspolitischer Koordinierung in der Währungsunion: politische oder ökonomische Logik?
}

\author{
von Armin Schäfer
}

In der Wirtschafts- und Währungsunion bestehen unterschiedliche wirtschaftspolitische Koordinierungsverfahren nebeneinander. Was erklärt die Unterschiede zwischen ihnen? Die politikwissenschaftliche Europaforschung gibt hierzu keine befriedigende Antwort, zumal sie sich in den vergangenen Jahren vor allem auf „weiche“ Koordinierung konzentriert hat und zur Erklärung der Wahl verbindlicher Verfahren auf die Argumente wirtschaftspolitischer Akteure - Europäische Zentralbank und Kommission - zurückgreift. Dieser Beitrag zeigt jedoch, dass deren Begründung nicht restlos überzeugen kann, da sich ihre Argumente nicht nur an ökonomischen Erfordernissen ausrichten, sondern auch aus dem Misstrauen gegenüber diskretionären politischen Entscheidungen speisen. Abschließend werden einige Überlegungen vorgestellt, wie eine politikwissenschaftliche Erklärung variierender Vergemeinschaftung anzulegen ist.

Within the EMU, different procedures of economic policy coordination co-exist. What accounts for their different degree of legalisation? Surprisingly, political scientists do not offer a convincing answer to this question as they have, until recently, been preoccupied with soft law procedures, often relying on the arguments put forward by supranational actors - mainly those of the European Central Bank and the Commission. This contribution, in contrast, seeks to show that these actors also fail to convincingly explain the choice of different coordination devices. Rather than relying on economic reasoning alone, many arguments reveal a general distrust of politics.

\section{Einleitung}

Die Europäische Union verfügt in der Wirtschaftspolitik über eine Reihe abgestufter Verfahren, mit denen sie die Abstimmung zwischen den Mitgliedstaaten, zwischen diesen und den EU-Institutionen sowie zwischen einzelnen Politikbereichen sicherstellen möchte. Diese Verfahren unterscheiden sich sowohl hinsichtlich ihrer Zentralisierung als auch im Grad rechtlicher Verbindlichkeit. Sie reichen von der Vergemeinschaftung der Geldpolitik bis zur sanktionslosen Koordinierung nationaler Arbeitsmarktpolitik. Daneben bestehen einige Politikfelder, in denen Konkurrenz dominiert und keine Zusammenarbeit institutionali- 
siert wurde (vgl. Tabelle 1). Wie lässt sich diese Mischung unterschiedlicher Abstimmungsinstrumente erklären? Die Europäische Zentralbank (EZB) und die Europäische Kommission beantworten diese Frage mit dem Hinweis auf die Vorteile einer stabilitätsorientierten Wirtschaftspolitik. Aus ihrer Sicht hat der Maastrichter Vertrag ein effizientes assignment geschaffen, das die Verantwortung für Preisstabilität, Wirtschafts- und Beschäftigungswachstum klar zuordnet. Erfüllen die Verantwortlichen - Zentralbank, Regierungen und Gewerkschaften - ihre Aufgaben gemäß dieser Zuordnung, entsteht ein makroökonomisch günstiger policy mix. Am wirksamsten wird dieses Ziel durch eine unabhängige Zentralbank und eine regelgebundene Fiskalpolitik erreicht. Klare Verhaltensregeln verhindern das zeitinkonsistente Verhalten wirtschaftspolitischer Akteure. ${ }^{1}$ Da die Arbeitslosigkeit nur über die Flexibilisierung des Arbeitsmarkts gesenkt werden kann und dies in der Verantwortung der Regierungen liegt, sind auf diesem Feld europäische Kompetenzen nicht notwendig. Der vorliegende Beitrag untersucht, ob diese Begründung für die beobachteten Unterschiede in den Verfahren wirtschaftspolitischer Koordinierung trägt.

Tabelle 1: Formen wirtschaftspolitischer Koordinierung in der EU

\begin{tabular}{|l|l|l|}
\hline Form der Abstimmung & Beispiel & $\begin{array}{l}\text { Verrechtlichung/ } \\
\text { Vergemeinschaftung }\end{array}$ \\
\hline \multirow{2}{*}{ Konkurrenz } & Lohnpolitik & gering \\
\cline { 1 - 2 } & Steuerpolitik & \\
\cline { 1 - 2 } $\begin{array}{l}\text { Kooperation } \\
\text { möglichkeit } \\
\text { (weiche Koordinierung) }\end{array}$ & Soziale Inklusion & $\begin{array}{l}\text { züge der Wirtschaftspolitik } \\
\text { (Art. 98-10o EGV) }\end{array}$ \\
\cline { 1 - 2 } $\begin{array}{l}\text { Koordinierung mit (politischer) } \\
\text { Sanktionsmöglichkeit }\end{array}$ & $\begin{array}{l}\text { Haushaltspolitik } \\
\text { (Art. 104 EGV, SWP) }\end{array}$ & \\
\hline $\begin{array}{l}\text { Koordinierung mit delegierter } \\
\text { Sanktionsmöglichkeit } \\
\text { (Gemeinschaftsmethode) }\end{array}$ & $\begin{array}{l}\text { Wettbewerbspolitik } \\
\text { (Kartell- und Beihilfenrecht) } \\
\text { (Art. 81-89 EGV) }\end{array}$ & \\
\hline Integration & Geldpolitik (Art. 105-112 EGV) & hoch \\
\hline
\end{tabular}

Quelle: Eigene Darstellung.

1 Issing, O. et al:: Monetary Policy in the Euro Area, Cambridge, 2001, 33. 
Der oben genannten Begründung für die Wahl unterschiedlicher Koordinierungsinstrumente stellt die Politikwissenschaft keine eigene Erklärung entgegen, weil sich die EU-Forschung fast ausschließlich auf die Analyse „weicher“ Verfahren konzentriert. ${ }^{2}$ Die Literatur zur Europäischen Beschäftigungsstrategie und zu der aus ihr hervorgegangenen Offenen Methode der Koordinierung (OMK) untersucht nur selten den Zusammenhang zwischen diesen Verfahren und der Währungsunion. Falls dies dennoch geschieht, wird argumentiert, dass die unverbindliche Koordinierung nationaler Arbeitsmarktreformen den Unterschieden nationaler Wohlfahrtsstaaten geschuldet sei. In den Kernbereichen nationaler Wohlfahrtsstaaten sei Harmonisierung oder rechtsverbindliche Koordinierung weder wünschenswert noch möglich. ${ }^{3}$ Stattdessen beschäftigen sich die meisten Autoren mit den Auswirkungen der OMK, d. h. insbesondere, ob es gelingt, über Lernprozesse nationale Reformen anzustoßen. Dies folgt einem jüngeren Trend innerhalb der Europaforschung, vor allem nach Funktionsweise und Folgen des Regierens im europäischen Mehrebenensystem zu fragen, und weniger die zuvor lange dominierende Frage nach den Integrationsursachen zu behandeln.

Die Selbstbeschränkung auf die Offene Methode der Koordinierung ist für die Politikwissenschaft aus zwei Gründen attraktiv. Zum einen erweisen sich zentrale Elemente weicher Koordinierung als in hohem Maß anschlussfähig gegenüber demokratietheoretischen Überlegungen. Die Offene Methode der Koordinierung ersetzt aus dieser Sicht hierarchische Steuerung durch deliberative Prozesse wechselseitigen Austauschs sowie Zwang durch Freiwilligkeit. Einige Autoren sehen darin eine neue Form des demokratischen Experimentalismus. ${ }^{4}$ Gerade die Abwesenheit von Sanktionen ermöglicht offene, lösungsorientierte Diskussionen. Der zweite Grund für die Attraktivität der neuen Koordinierungsverfahren ist, dass mit der Europäischen Beschäftigungsstrategie und der OMK die soziale Dimension des Europäisierungsprozesses aufgewertet wurde. Beherrschten lange Zeit wirtschaftliche Themen die europäische Integration, werden diese seit dem Lissabonner Gipfel im März 2000 durch das erklärte Ziel ergänzt, wirtschaftliche Effizienz mit sozialem Ausgleich in Einklang zu bringen. Die OMK soll die not-

2 Zentrales Unterscheidungsmerkmal zwischen harter und weicher Koordinierung sind rechtsverbindliche Sanktionen. Siehe Abbott, K. W./Snidal, D.: Hard and Soft Law in International Governance, in: International Organization, 54/3 (2000), 421-456.

3 Borrás, S./Jacobsson, K.: The Open Method of Co-ordination and New Governance Patterns in the EU, in: Journal of European Public Policy, 11/2 (2004), 185-208, hier $190 \mathrm{f}$.

4 Zeitlin, J.: Introduction: Governing Work and Welfare in a New Economy: European and American Limits, in: Zeitlin, J./Trubek, D. M. (Hg.): Governing Work and Welfare in a New Economy. European and American Experiments, Oxford, 2003, 1-30, hier 5; Eberlein, B./Kerwer, D.: Theorising the New Modes of European Union Governance, in: European Integration online Papers, 6/15 (2002). 
wendige Reform der sozialen Sicherung mit dem Europäischen Sozialmodell versöhnen, das auf Solidarität und Teilhabe der Sozialpartner beruht. Diese Strategie unterscheidet sie von den „neoliberalen Vorschlägen“ der OECD zur Arbeitsmarktreform. ${ }^{5}$

Diese beiden Sichtweisen lassen sich nur aufrechterhalten, wenn die OMK von der Währungsunion getrennt betrachtet wird, die sich gerade dadurch auszeichnet, dass die Entscheidungen der EZB und die fiskalpolitischen Regeln dem diskretionären Zugriff entzogen sind. Darüber hinaus beschränkt die Wirtschaftsund Währungsunion die inhaltliche Reichweite der OMK. So kann, wie Scharpf hervorhebt, mit ihr etwa nicht beschlossen werden, auf steigende Arbeitslosigkeit mit niedrigeren Zinsen zu reagieren, einzelne Staaten zeitweise von den Sparzwängen des Stabilitäts- und Wachstumspakts (SWP) zu befreien, bei steigenden Gesundheitskosten Positivlisten für Medikamente einzuführen, eine Mindestbesteuerung festzulegen oder die Kapitalmobilität zu beschränken. ${ }^{6}$ Der effektive Spielraum für Deliberation beschränkt sich somit auf die angebotsseitige Reform des Arbeitsmarkts. Zudem wird der Unterschied zwischen der OECD und der EU in der Literatur überzeichnet. Tatsächlich stimmen beide Organisationen in ihren arbeitsmarktpolitischen Empfehlungen weitgehend überein. Die Grundzüge der Wirtschaftspolitik empfehlen Deutschland die Dezentralisierung der Lohnverhandlungen, Differenzierung der Löhne, die Steigerung der Anreize zur Arbeitsaufnahme und kritisieren insgesamt die starke Regulierung der Arbeitsmärkte. ${ }^{7}$

Die einseitige Konzentration auf weiche Koordinierungsverfahren führt aber nicht nur zur verzerrten Bewertung der OMK, sondern versperrt der politikwissenschaftlichen EU-Forschung auch die Möglichkeit, nach den Ursachen für die ungleiche Vergemeinschaftung und nach dem Zusammenspiel verschiedener Koordinierungsinstrumente zu fragen. ${ }^{8}$ Diese Selbstbeschränkung hat zur Folge, dass die Politikwissenschaft die ökonomische Begründung der gegenwärtigen Aufgabenzuteilung im Rahmen der Währungsunion übernimmt. Im Folgenden wird der Frage nachgegangen, ob diese Begründung plausibel ist. Dazu wird in einem ersten Schritt die Logik des Maastricht Assignment dargestellt. Grundlage

5 So Casey, B. H.: The OECD Jobs Strategy and the European Employment Strategy, in: European Journal of Industrial Relations, 10/3 (2004), 329-352.

6 Scharpf, F. W.: The European Social Model: Coping with the Challenges of Diversity, in: Journal of Common Market Studies 40/4 (2002), 645-670, hier 655.

7 Europäische Kommission: Empfehlungen der Kommission zu den Grundzügen der Wirtschaftspolitik der Mitgliedstaaten und der Gemeinschaft (im Zeitraum 2003-2005), KOM 170 (2003), 25-28.

8 Siehe aber Hodson, D.: Macroeconomic Co-ordination in the Euro-Area: the Scope and Limits of the Open Method, in: Journal of European Public Policy, 11/2 (2004), 231-248; Trubek, D.M./Mosher, J. S.: New Governance, Employment Policy, and the European Social Model, in: Zeitlin, J./Trubek D. M. (Hg.), a. a. O., 33-58, hier $34-38$. 
hierfür sind die Veröffentlichungen und Politikempfehlungen von EZB und Kommission. In einem zweiten Schritt wird gezeigt, dass das Maastrichter Modell wirtschaftspolitischer Koordinierung nicht frei von Widersprüchen ist. Weder die zugrunde liegenden Annahmen noch die Wirtschaftsdaten der vergangenen Jahre belegen stichhaltig, dass die bestehende Koordinierung einen vorteilhaften policy mix gewährleistet. Analysiert man die Argumente von EZB und Kommission, erscheint die Aufgabenteilung eher als Ausdruck des Misstrauens gegenüber einem zu großen politischen Entscheidungsspielraum denn als sachlogisch begründet. Im letzten Abschnitt soll skizziert werden, wie eine politikwissenschaftliche Erklärung aussehen könnte. Sie stellt vor allem auf den Widerstand nationaler Regierungen gegen weit gehende Souveränitätstransfers ab.

Zwei Einschränkungen müssen vorab genannt werden. Weder eine vollständige Erklärung unterschiedlicher Vergemeinschaftung noch ein überlegenes Modell wirtschaftspolitischer Koordinierung sind Ziel dieses Aufsatzes. Stattdessen soll der politische Kern der ökonomischen Argumentation von EZB und Kommission verdeutlicht werden.

\section{Koordinierung durch Aufgabentrennung: das Maastricht Assignment}

Der Übergang zur Wirtschafts- und Währungsunion wirft die Frage auf, wie die Entscheidungen der verschiedenen wirtschaftspolitischen Akteure aufeinander abgestimmt werden können. Dabei folgt möglicher Koordinierungsbedarf aus der Vergemeinschaftung der Geldpolitik bei weiterhin nationalstaatlicher Zuständigkeit für die öffentlichen Haushalte, Arbeits- und Produktmärkte sowie die Lohnpolitik. Für einen positiven policy mix müssen sowohl die Politik der Mitgliedstaaten und einzelne Politikfelder miteinander als auch diese zusammengenommen mit der Geldpolitik vereinbar sein. Koordinierung dient zum einen der Produktion öffentlicher Güter, beispielsweise einer niedrigen Inflationsrate, zum anderen soll sie negative Externalitäten vermeiden. ${ }^{9}$

Koordinierung kann vorab durch die Zielabstimmung zwischen den Akteuren stattfinden oder implizit und ex post erfolgen. Die Währungsunion folgt der zweiten Variante, wobei die Zentralbank als Garant einer stabilitätsorientierten Politik fungiert. Die Unabhängigkeit der EZB erhöht ihre Fähigkeit und Glaub-

9 European Commission, Directorate-General for Economic and Financial Affairs: Co-ordination of Economic Policies in the EU: A Presentation of Key Features of the Main Procedures, Euro Papers, 45, Brüssel, 2002, 3-4. 
würdigkeit, niedrige Inflationsraten auch gegen Widerstand durchzusetzen. Da sie andere Ziele nur in Betracht zieht, solange diese die Preisstabilität nicht gefährden, liegt die Verantwortung für Wirtschafts- und Beschäftigungswachstum bei den Regierungen und den Gewerkschaften. Letztgenannte können nur dann ein positives Zusammenspiel mit der Geldpolitik erreichen, wenn sie die Strategie der EZB in ihren Entscheidungen berücksichtigen. Indem die Zentralbank die Erwartungen der Wirtschaftsakteure hinsichtlich der künftigen Inflationsentwicklung lenkt, beeinflusst sie deren Lohn- und Preissetzungsverhalten. Koordinierung wird nicht durch Zusammenarbeit, sondern durch Aufgabentrennung erreicht:

„Genießt eine Zentralbank ein hohes Maß an Glaubwürdigkeit bei der Verfolgung ihres Ziels, kann die Geldpolitik einen starken Einfluss auf die Preisentwicklung ausüben, indem sie die Erwartungen der Wirtschaftsakteure hinsichtlich der künftigen Inflationsentwicklung lenkt und damit deren Lohn- und Preissetzungsverhalten beeinflusst. Das Vertrauen darauf, dass eine Zentralbank Preisstabilität dauerhaft gewährleistet, spielt dabei eine entscheidende Rolle. " ${ }^{10}$

Das beste Ergebnis wird erzielt, wenn jeder Akteur die ihm zugeordnete Aufgabe erfüllt. Eine klare Aufgabenaufteilung (assignment), bei der die Zentralbank ausschließlich für das Preisniveau, die Regierung für Strukturreformen, Haushaltskonsolidierung und verlässliche Rahmenbedingungen und die Gewerkschaften über die Lohnpolitik für das Beschäftigungswachstum verantwortlich sind, ist die Voraussetzung für eine erfolgreiche Wirtschaftspolitik. ${ }^{11}$ Die Aufgaben der Akteure zu vermischen, führt dagegen zu wenig wünschenswerten Ergebnissen. Der Unabhängigkeit der Zentralbank kommt überragende Bedeutung zu, weil nur sie auf die politischen Entscheidungen der anderen Wirtschaftsakteure disziplinierend einwirken kann. Aus dieser Sicht ist weitere wirtschaftspolitische Koordinierung nicht notwendig oder sogar schädlich, da sie den Standortwettbewerb entschärft und nötige Strukturreformen sowie eine beschäftigungsfördernde Lohnspreizung verhindert.

Die dem Maastrichter assignment zugrunde liegenden Annahmen lassen sich wie folgt zusammenfassen: ${ }^{12}$

- Die langfristige Neutralität des Geldes: Veränderungen der Geldmenge schlagen sich in einer Änderung des allgemeinen Preisniveaus nieder, ziehen aber

10 Europäische Zentralbank: Die Geldpolitik der EZB, Frankfurt, 2004, 47.

11 Caesar, R./Kösters, W.: Europäische Wirtschafts- und Währungsunion: Europäische Verfassung versus Maastrichter Vertrag, in: Integration, 27/4 (2004), 288-300, hier 291.

12 Für eine Darstellung und Diskussion des Maastrichter assignment aus post-keynesianischer Sicht siehe Hein, E./Truger, A.: Makroökonomische Koordinierung als wirtschaftspolitisches Konzept, in: Hein, E. u. a. (Hg.): Europas Wirtschaft gestalten, Hamburg, 2004, 15-50, hier $18 \mathrm{f}$. 
keine anhaltende Veränderung der gesamtwirtschaftlichen Produktion oder der Arbeitslosigkeit nach sich. ${ }^{13}$

- Die Fiskalpolitik hat langfristig keinen Einfluss auf Wachstum und Beschäftigung, sie sollte stattdessen zur Preisstabilität beitragen. Die Fiskalpolitik hat sich in der Vergangenheit als ungeeignet erwiesen, den Konjunkturzyklus zu steuern. Regierungen sollten daher auf diskretionäre Maßnahmen verzichten und über den Konjunkturzyklus einen ausgeglichen Hauhalt anstreben, während die automatischen Stabilisatoren greifen. ${ }^{14}$

- Die Höhe der Arbeitslosigkeit wird durch die Flexibilität des Arbeitsmarktes und den Umfang der verfügbaren Lohnersatzleistungen bestimmt. ${ }^{15}$

- Die Lohnentwicklung wirkt sich kurzfristig auf die Inflation und langfristig auf die Höhe der Arbeitslosigkeit aus.

Die EZB vertritt diese Positionen regelmäßig in ihren Veröffentlichungen, in den Stellungnahmen zur Politik der Mitgliedstaaten und in der Diskussion um eine Reform des Stabilitäts- und Wachstumspaktes. ${ }^{16}$ Sie bilden aber auch die Grundlage für die Grundzüge der Wirtschaftspolitik. ${ }^{17}$ Wird hierbei von Koordinierung gesprochen, bezieht sich dies auf das Modell der ex post-Koordinierung, bei dem die Wirtschaftsakteure den Vorgaben der Zentralbank folgen. Wenn die Kommission eine stärkere Koordinierung fordert, klagt sie die konsequente Anwendung bestehender Regeln ein, nicht weiter gehende Absprachen zwischen den Akteuren. Unmissverständlich ist die Ablehnung politischer Zielabstimmung durch das EZB-Präsidiumsmitglied Otmar Issing:

„To put it simply, an efficient initial assignment of objectives and instruments, together with a clear division of responsibilities, will achieve implicitly co-ordinated outcomes ex post and thereby largely substitute the need for explicitly co-ordinated policies later on. In the euro area, the Maastricht Treaty already provides for such an efficient assignment of objectives with a sound and clear allocation of responsibilities to the individual policy-makers, thus making the scope for additional policy co-ordination rather limited. This assignment clearly defines the role of the European Central Bank (ECB) and provides rules for the other policy-makers' contributions to economic stability. " 18

\section{Europäische Zentralbank, a. a. O., 41.}

European Central Bank: The Operation of Automatic Fiscal Stabilisers in the Euro Area, in: ECB Monthly Bulletin, 4/2002, 33-46, hier 36 f.; dies.: Fiscal Policy Influences on Macroeconomic Stability and Prices, in: ECB Monthly Bulletin, 4/2004, 45-57, hier 52.

15 Blanchard, O./Katz, L.F.: What We Know and Do Not Know About the Natural Rate of Unemployment, in: Journal of Economic Perspectives, 11/1 (1997), 51-72.

Siehe European Central Bank: The Outcome of the ECB's Evaluation of its Monetary Policy Strategy, in: ECB Monthly Bulletin, 6/2003, 79-92.

Europäische Kommission: Empfehlungen, a. a. O., 5-7.

Issing, O.: On Macroeconomic Policy Co-ordination in EMU, in: Journal of Common Market Studies, 40/2 (2002), 345-358, hier 348 . 
Die privilegierte Stellung der Zentralbank macht im Zusammenspiel mit der regelgebundenen Fiskalpolitik sowohl weitere Koordinierung als auch zusätzliche Kompetenztransfers auf die europäische Ebene überflüssig. Ausgehend von dieser Annahme konnte ein institutionell schlankes Modell der Währungsunion gewählt werden, das sich von früheren Entwürfen durch den geringen Souveränitätstransfer unterscheidet. Im Gegensatz dazu hatten noch der Werner-Bericht (1970) und der MacDougall-Bericht (1977) betont, dass eine Währungsunion sowohl die Zentralisierung der Entscheidungen über die nationalen Haushalte als auch die Ausweitung des Gemeinschaftsbudgets notwendig mache. In den Schlussfolgerungen des Werner-Berichts wurde daher neben der Zentralbank auch eine europäische Wirtschaftsregierung gefordert. ${ }^{19}$ Erst mit der Abkehr vom Keynesianismus entfiel das Motiv einer nach Maßgabe der Gemeinschaftsziele harmonisierten Haushaltspolitik. Anstelle der Stabilisierung der aggregierten Nachfrage wurde im Vorlauf zu Maastricht der Haushaltspolitik die Aufgabe zugeschrieben, das Inflationsziel der Geldpolitik zu unterstützen. Der Delors-Bericht (1989) sah folglich die Festlegung wirksamer Obergrenzen für Haushaltsdefizite vor. Dafür wurden verbindliche, vertraglich festgelegte Regeln gefordert, während der gemeinschaftsweite policy mix über die weiche Koordinierung nationaler Politiken geregelt werden sollte. Eine europäische Wirtschaftsregierung war indessen nicht mehr vorgesehen. Der Verzicht auf eine umfangreiche wirtschaftspolitische Koordinierung war möglich, weil angenommen wurde, dass kein Zielkonflikt zwischen einer stabilitätsorientierter Geldpolitik und Wirtschaftswachstum bestand.

Der Vorteil einer institutionell weniger anspruchsvollen Konzeption der Währungsunion lag darin, die Zustimmung der Regierungen zu erleichtern. Ob sie tatsächlich auch zu einer reibungslosen ex post-Koordinierung führt, soll im nächsten Abschnitt untersucht werden.

19 „Die Wirtschafts- und Währungsunion bringt es mit sich, daß die wichtigsten wirtschaftspolitischen Entscheidungen auf Gemeinschaftsebene getroffen werden und infolgedessen die erforderlichen Befugnisse von nationaler Ebene auf die Ebene der Gemeinschaft übertragen werden. [...] Auf institutioneller Ebene erscheinen in der Endphase zwei Gemeinschaftsorgane erforderlich: ein wirtschaftspolitisches Entscheidungsgremium und ein gemeinschaftliches Zentralbankensystem" (Werner, P.: Bericht an Rat und Kommission über die stufenweise Verwirklichung der Wirtschafts- und Währungsunion in der Gemeinschaft, in: Krägenau, H./Wetter, W. (Hg.): Europäische Wirtschafts- und Währungsunion, BadenBaden, 1993, 98-106, hier 105). 


\section{Horizontale, vertikale und zeitliche Abstimmungsprobleme im Rahmen der Währungsunion}

Im Folgenden werden drei Probleme des Maastrichter assignment behandelt: das Übergangs-, das signaling- sowie das Korrespondenzproblem. Der nächste Abschnitt argumentiert, dass die Prämissen, auf denen das Modell einer impliziten Koordinierung aufbaut, unzulänglich erfüllt sind und dass die Währungsunion in Abwesenheit dieser Voraussetzungen den Übergang zu einem neuen Gleichgewicht erschwert. Danach wird das Problem der Asymmetrie zwischen Vergemeinschaftung der Geldpolitik und relativer Dezentralisierung der Wirtschaftspolitik behandelt. Schließlich wird zu fragen sein, ob sich die Aufteilung in weiche und harte Koordinierungsverfahren schlüssig aus der wirtschaftspolitischen Argumentation ergibt.

\section{Das Übergangsproblem}

Aus der aktuellen Diskussion um den Stabilitäts- und Wachstumspakt (SWP) wurde deutlich, dass in der Fiskalpolitik Übergangsprobleme von hohen Defiziten zu mittelfristig ausgeglichenen Haushalten vorhanden sind. Sie erklären sich aus der Inkongruenz der im Maastrichter Vertrag festgelegten Konvergenzkriterien einerseits und des SWP andererseits - und dies obwohl der SWP die Anwendung des Verfahrens bei übermäßigen Defiziten regeln sollte. Der damaligen Bundesregierung erschien es erforderlich, dem Defizitverfahren durch die Festlegung quasi-automatischer Anwendungsregeln Härte zu verleihen, weil der vorhandene Sanktionsmechanismus zu viel Raum für politische Entscheidungen und Kompromisse ließ. ${ }^{20}$

Der SWP beinhaltet zwei Kernelemente. Zum einen formuliert er das im Vergleich zu den Konvergenzkriterien anspruchsvollere Ziel eines mittelfristig ausgeglichenen oder einen Überschuss aufweisenden Haushalts. Zum anderen soll über ein Frühwarnsystem vor der Gefahr übermäßiger Defizite gewarnt werden. Das Übergangsproblem der Haushaltspolitik besteht darin, dass der SWP davon ausgeht, die Mitgliedstaaten hätten bereits das Ziel nahezu ausgeglichener Haushalte erreicht. ${ }^{21}$ Dagegen definieren die Konvergenzkriterien ein Haushalts-

Costello, D.: The SGP: How Did We Get There?, in: Brunila, A./Buti, M./Franco, D. (Hg.): The Stability and Growth Pact, Basingstoke, 2001, 106-136, hier 106; Stark, J.: Genesis of a Pact, in: Brunila, A./Buti, M./ Franco, D. (Hg.), a.a.O., 77-105, hier $81 \mathrm{f}$.

21 Buti, M./Eijffinger, S./Franco, D.: Revisiting the Stability and Growth Pact: Grand Design or Internal Adjustment?, European Economy. Economic Papers, 180, Brüssel, 2003, $5 \mathrm{f}$. 
defizit von drei Prozent als Grenzwert, der zur Teilnahme an der Währungsunion berechtigt. Da die Entscheidung, welche Staaten die wirtschaftlichen Voraussetzungen für die Währungsunion erfüllten, sich zwar an den Konvergenzkriterien orientierte, aber im Kern eine politische blieb, nahmen Staaten teil, die das Verschuldungskriterium nicht erfüllten und/oder nahe an der Defizitgrenze von drei Prozent lagen. Die heutigen Probleme des SWP ergeben sich dementsprechend aus der Diskrepanz zwischen der politischen Logik der Konvergenzkriterien, die eine breite Mitgliedschaft in der Währungsunion anstrebte, und der ökonomischen des Stabilitätspakts.

Mit der Zulassung zur Währungsunion waren die Funktionsvoraussetzungen des Stabilitätspakts nicht erfüllt, und weil in der Folge weder die vorhandenen Instrumente noch der politische Wille zur Durchsetzung der Haushaltskonsolidierung ausreichten, überschritten einige Staaten (Portugal, Deutschland, Frankreich) im nächsten Abschwung das Defizitkriterium. Ausgehend von einem deutlichen Defizit befinden sich diese Staaten nun im permanenten Konflikt mit dem SWP, welcher sie - strikt eingehalten - zwänge, eine in der Tendenz prozyklische Haushaltspolitik zu betreiben, in der die automatischen Stabilisatoren nicht in vollem Umfang greifen könnten. Damit kann der SWP die Wachstumskrise verschärfen und den Übergang zu ausgeglichenen Haushalten verhindern, die jedoch die Voraussetzung für seine Anwendung sind. Aus Abbildung 1 wird ersichtlich, dass sich für Italien, Portugal, Frankreich und Deutschland die Haushaltsposition gegenüber 1998 verschlechtert hat bzw. der Abstand zu den Vorgaben des SWP vergrößert hat. Dagegen weisen Österreich, Spanien und Belgien eine deutlich bessere Bilanz auf, obwohl auch sie 1998 noch Defizite von über zwei Prozent hatten.

In der Reformdiskussion um den SWP schlägt die Europäische Kommission eine Modifizierung der Regeln vor. ${ }^{22}$ Zukünftig sollen die Mitgliedstaaten in Aufschwungphasen zur Haushaltskonsolidierung gezwungen werden. Damit soll der Asymmetrie des Pakts, der nur im Abschwung greift, begegnet werden. ${ }^{23}$ Dies könnte in Zukunft eine größere Kongruenz zwischen dem SWP und den Ausgangsbedingungen in den Euro-Staaten bewirken. Allerdings konnten sich die stärkung der haushaltspolitischen Koordinierung, KOM 668 (2002), Brüssel.

23 Die Defizitregeln wirken auch insofern asymmetrisch, als Mitgliedstaaten, die eine expansive, inflationsfördernde Haushaltspolitik betreiben, ohne jedoch die Defizitgrenze zu erreichen, nicht diszipliniert werden können. Für diesen Fall sind keine rechtsverbindlichen Sanktionen vorgesehen. Stattdessen muss über die Grundzüge der Wirtschaftspolitik politischer Druck auf die Regierung des betreffenden Lands ausgeübt werden. Eine solche Konstellation führte zu der Rüge Irlands durch den Ministerrat im Jahr 2001. 
Regierungen bisher nicht auf eine Reform des Stabilitäts- und Wachstumspakts einigen, die die Haushaltskonsolidierung für Ab- und Aufschwungphasen verbindlich regelt.

Abbildung 1: Defizite und Überschüsse ausgewählter Euro-Staaten in \% des BIP

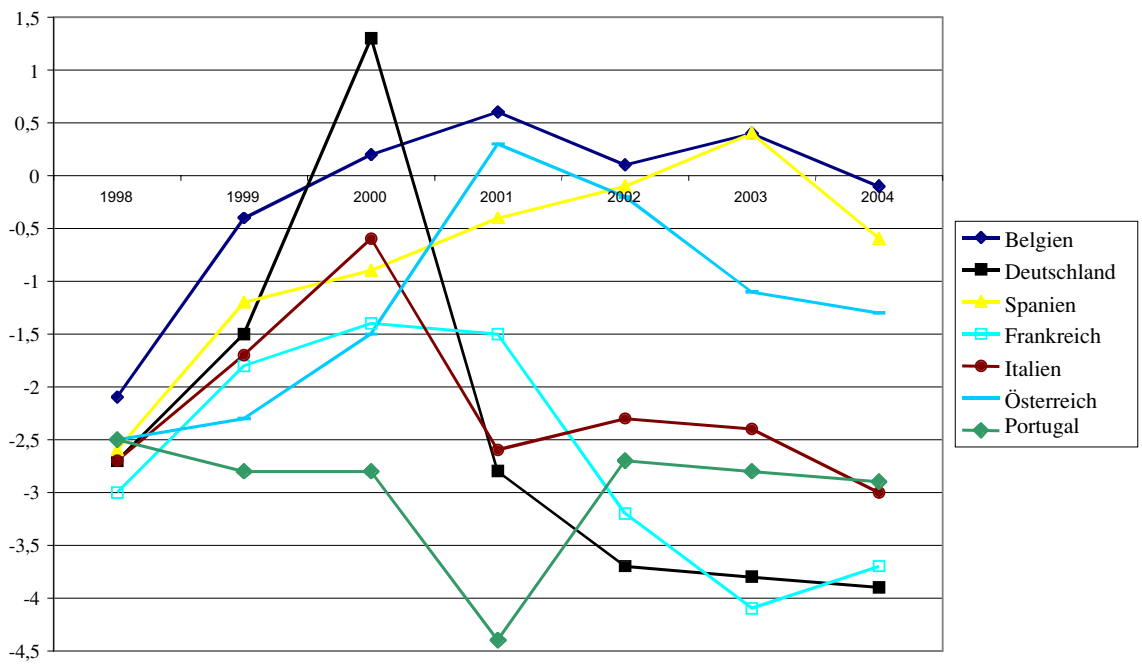

Quelle: Eurostat.

Neben der Differenz zwischen den Defizitregeln des EG-Vertrags und dem SWP ist für das Übergangsproblem die vertikale Abstimmung von Geld- und Fiskalpolitik entscheidend. Nach dem Maastrichter assignment liegt die Verantwortung für den Ausgleich symmetrischer Schocks, die den gesamten Währungsraum betreffen, vorwiegend bei der Zentralbank. Asymmetrische Schocks müssen dagegen durch die automatischen Stabilisatoren und die Lohn- und Arbeitsmarktpolitik der einzelnen Länder aufgefangen werden. Dies setzt voraus, dass die EZB eine Mitverantwortung für die konjunkturelle Entwicklung der Euro-Zone übernimmt. Eine Schwierigkeit dieser Aufteilung liegt im unterschiedlichen Zeithorizont der Wirtschaftsakteure. ${ }^{24}$ Die EZB kann verzögert auf einen Abschwung reagieren, um sicherzustellen, dass keine Inflationsgefahr besteht. Dabei erhöht ge-

24 Das ist ein Problem, weil die Neutralität des Geldes nur mittelfristig gilt. Aufgrund eigener Modellrechnungen kommt die EZB (Die Geldpolitik, a. a. O., 49) zu folgendem Schluss: „Ein Anstieg der kurzfristigen Zinssätze führt zu einer vorübergehenden Verringerung der Produktion, die etwa zwei Jahre nach dem eigentlichen geldpolitischen Impuls ihre negative Spitze erreicht und anschließend auf ihr ursprüngliches Niveau zurückkehrt." Das heißt, kurzfristig hat die Geldpolitik einen merklichen Einfluss auf das Wachstum. 
rade die Unbestimmtheit der Zwei-Säulen-Strategie (wirtschaftliche und monetäre Analyse) ihren diskretionären Spielraum. So hat die EZB später und weniger energisch mit Zinssenkungen als die Federal Reserve auf den wirtschaftlichen Einbruch 2001 reagiert, ${ }^{25}$ und dies obwohl der Abschwung wesentlich durch externe, symmetrisch wirkende Faktoren ausgelöst wurde (Terrorismus, Ölpreis, Weltkonjunktur). Zudem ist die realwirtschaftliche Entwicklung nur ein Ziel der EZB. Da ihre Strategie mittelfristig ausgelegt ist, ging es in den ersten Jahren auch immer um die Demonstration der eigenen Glaubwürdigkeit. Die zahlreichen Schocks zwischen 1999 und 2004 stellten für die EZB vor allem eine Herausforderung dar, die Inflationserwartung gering zu halten und eine dauerhafte Lohn-Preis-Spirale zu verhindern. ${ }^{26}$

Aber auch ohne externe Schocks bleibt unklar, wie der Übergang von einem Gleichgewicht mit relativ hohen (Real-)Zinsen und hohen Defiziten zu einem mit jeweils niedrigen Werten bewerkstelligt werden kann. Vor allem für Regierungen entstehen bei der Defizitreduzierung Kosten, etwa durch eine schneller steigende Arbeitslosigkeit, die bei einem kurzfristigen, durch den Wahlzyklus bestimmten Zeithorizont prohibitiv hoch sein können. ${ }^{27}$ Drohen die elektoralen Kosten für eine Regierung so hoch zu werden, dass sie die nächsten Wahlen verliert, kann die Akzeptanz höherer Defizite eine überlegene Strategie sein..$^{28}$ Abhängig davon, wie stark der politische Konjunkturzyklus die Entscheidung beeinflusst, kann gerade eine nicht-akkommodierende Zentralbankstrategie das gewünschte Ergebnis verhindern. Dieses Problem wird durch die nachrangige Wachstumsorientierung der Zentralbank im Vergleich zu ihrer Inflationsaversion verstärkt, die zu einem asymmetrischen Reaktionsmechanismus führt. Während sie unmittelbar auf eine gestiegene Inflationsgefahr antwortet, wird sie nur zögernd auf Konsolidierungsanstrengungen reagieren, um sicherzustellen, dass der eingeschlagene Pfad beibehalten wird. Sie verzichtet zumindest kurzfristig auf die Stabilisierung der durch eine restriktive Haushaltspolitik sinkenden aggregierten Nachfrage. Aber selbst eine symmetrische Reaktionsfunktion der Zentralbank - wenn also die EZB bei geringer Inflationsgefahr die Zinsen genauso stark senkt, wie sie sie bei drohender Inflation anhebt - kann durch die verzögerte Wirkung niedriger Zinsen sowie die von den anderen Wirtschaftsakteuren unterstellte asymmetrische

Hein, E./Truger, A., a. a. O., 22-25.

26 So die Bewertung der Europäischen Zentralbank. Siehe EZB: Die Geldpolitik, a. a. O., 105.

27 Vgl. hierzu Fußnote 24.

28 Die EZB argumentiert, dass Haushaltskonsolidierung nicht-keynesianische, expansive Effekte haben kann. Ein wesentlicher Effekt einer mittelfristig angelegten Konsolidierungsstrategie liegt darin, Vertrauen in das Regierungshandeln aufzubauen, das dann zu höheren Investitionen und Konsum führt. EZB: Die Geldpolitik, a. a. O., $48 \mathrm{f}$. 
Reaktionsfunktion ein positives Zusammenspiel von Geld- und Fiskalpolitik verhindern. ${ }^{29}$

Zusammengenommen bedeutet dies, dass ein vertikales Koordinierungsproblem zwischen den Mitgliedstaaten und der Zentralbank besteht, das sich nicht darauf reduzieren lässt, ob die Reaktionsfunktion der EZB angemessen ist oder nicht. Das Maastrichter assignment unterstellt ein mechanistisches Zusammenspiel der Wirtschaftsakteure. Sein Ziel besteht vornehmlich darin, willkürliche politische Entscheidungen zurückzudrängen. Allerdings gilt dies viel stärker für die Regierungen als für die Zentralbank selbst, deren Autonomie und geldpolitische Strategie ihr mehr Handlungsfreiheit gewähren. ${ }^{30}$

\section{Das signaling-Problem}

Bisher wurde unterstellt, es gäbe nicht nur eine zentralisierte Geldpolitik, sondern ebenfalls eine einheitliche Fiskalpolitik. Allerdings bewirkt der Übergang zur Währungsunion tatsächlich eine relative Dezentralisierung der Wirtschaftspolitik. Dadurch verschärfen sich einige der angesprochenen Probleme, da sich im Vergleich zum Nationalstaat die Adressaten der Geldpolitik vervielfachen. Damit die EZB die ihr zugeschriebene Aufgabe als stabilitätspolitischer Anker erfüllen kann, muss die Politik der Mitgliedstaaten aufeinander abgestimmt werden. Hier treten allerdings Probleme kollektiven Handelns auf. Die Geldpolitik kann nur die Konsolidierungsbemühungen der Mitgliedstaaten durch niedrigere Zinsen begleiten, wenn die Fiskalpolitik der Euro-Zone insgesamt restriktiv ist. Befinden sich die Mitgliedstaaten an unterschiedlichen Punkten des Konjunkturzyklus, werden die Zinsen für einige von ihnen zu hoch, für andere zu niedrig ausfallen. Da die Geldpolitik auf den gesamten Euro-Raum reagiert, muss eine hinreichend große Anzahl von Ländern über einen längeren Zeitraum Haushaltskonsolidierung verfolgen, um die EZB zu einer Zinssenkung zu bewegen. Idealer Weise sollten die Regeln des SWP die nationalstaatlichen Politiken koordinieren und somit ihre Glaubwürdigkeit gegenüber der EZB erhöhen. Da jedoch dessen Funktionsvoraussetzungen nicht erfüllt sind, brechen etliche Mitgliedstaaten den SWP. Diese Regelverstöße weisen aus Sicht der EZB darauf hin, dass den Regierungen der Wille zur Haushaltsdisziplin fehlt. Geld- und Fiskalpolitik unterstützen sich somit nicht in der gewünschten Weise.

30 
Wenn man den dargestellten Zusammenhang zwischen einer asymmetrischen Reaktionsfunktion der Zentralbank und der Schwierigkeit der Regierungen, die Glaubwürdigkeit ihrer Haushaltskonsolidierung zu demonstrieren, akzeptiert, wird deutlich, dass eine strikte Aufgabenteilung, die keine Kooperation zwischen den Akteuren oder gar eine Koordinierung ihrer Handlungen vorsieht, nicht automatisch optimale Ergebnisse produziert. Im Gegenteil, eine ausschließlich auf Preisstabilität ausgerichtete Zentralbank, die nicht die ökonomischen Folgen einer restriktiven Haushaltspolitik durch Zinssenkungen abfedert, kann dem Abbau von Defiziten im Weg stehen, da sie dessen politische Kosten für die Regierung erhöht. ${ }^{31}$

Analog lässt sich für die Koordinierung von Strukturreformen argumentieren. Wenngleich der Nutzen der Reformen überwiegend im eigenen Land anfällt, wirkt sich eine anhaltende Wachstumsschwäche in einem Mitgliedsland negativ auf die anderen Länder aus. Zumindest zum Teil ist auch das Wirtschaftswachstum in der Euro-Zone ein öffentliches Gut. Nun könnten Strukturreformen ebenfalls durch die Geldpolitik erleichtert werden, falls die EZB ein gestiegenes Wachstumspotenzial durch niedrigere Zinsen begleitete. ${ }^{32}$ Ähnlich wie in der Haushaltspolitik setzt dies ein konzertiertes Vorgehen der Regierungen voraus, das ihre Reformbereitschaft glaubhaft vermittelt. Sowohl in der Haushaltspolitik als auch bei Reformen der Arbeits-, Produkt- und Kapitalmärkte gibt es horizontalen (zwischenstaatlichen) und vertikalen (gegenüber der EZB) Abstimmungsbedarf. ${ }^{33}$

Die Absichtserklärungen des Europäischen Rates sowie die multilaterale Überwachung durch die Grundzüge der Wirtschaftspolitik haben die Funktion, die Regierungen wechselseitig auf die vereinbarten Ziele zu verpflichten und dies gegenüber der Öffentlichkeit sowie der Zentralbank zu dokumentieren. Die länderspezifischen und gemeinschaftlichen Politikempfehlungen in den Grundzügen sollen die Ernsthaftigkeit der Verpflichtung unterstreichen, indem sie überprüfbare, transparente Ziele vorgeben. Allerdings sind weder in den Grundzügen der Wirtschaftspolitik noch in der Europäischen Beschäftigungsstrategie Sanktionsmöglichkeiten vorhanden. Falls die Flexibilisierung der Arbeitsmärkte neben Preisstabilität und Haushaltskonsolidierung die dritte Säule des Maastrichter

31 Kasten und Soskice schlagen deshalb eine symmetrische Reaktionsfunktion der EZB vor, bei der sie solange die Inflationsrate sich innerhalb der Zielzone befindet, ihren zinspolitischen Spielraum nutzt, um das Wirtschaftswachstum zu fördern. Kasten, G./Soskice, D.: Möglichkeiten und Grenzen von Beschäftigungspolitik in der Europäischen Wirtschafts- und Währungsunion, WZB Discussion Paper, 303, Berlin, 1999, 43.

32 Allsopp, C./Vines, D.: The Assessment: Macroeconomic Policy After EMU, in: Oxford Review of Economic Policy, 14/3 (1998), 1-23, hier 7.

33 Jacquet, P./Pisani-Ferry, J.: Economic Policy Co-ordination in the Euro-Zone, London, 2001. 
assignment und Voraussetzung für das Funktionieren der Währungsunion sein soll, ${ }^{34}$ stellt sich die Frage, weshalb hier ein weiches Koordinierungsverfahren gewählt wurde. Im Gegensatz zum Nationalstaat ist die EZB nicht zur gezielten Bestrafung möglichen Fehlverhaltens einzelner Regierungen (oder der Sozialpartner) in der Lage. In der Wirtschaftspolitik kommt diese Aufgabe dem Gruppendruck (peer pressure) der Betroffenen zu. Während in der Fiskalpolitik Sanktionen notwendig erschienen, um zeitinkonsistentes Verhalten der Regierungen $\mathrm{zu}$ vermeiden, führt dieselbe Logik nicht zu Sanktionen bei einer Wirtschaftspolitik, die „das ordnungsgemäße Funktionieren der Wirtschafts- und Währungsunion zu gefährden droht“" (Art. 99 Abs. 4 EGV).

Besonders schwierig gestaltet sich die Koordinierung der Lohnpolitik, da hier Konkurrenz zwischen den Mitgliedstaaten dominiert (siehe oben Tabelle 1). Die Frage, ob und wie die Lohnpolitik koordiniert werden sollte, ist auch deshalb schwierig zu beantworten, weil der Koordinierungsbedarf unterschiedliche (gegensätzliche) Ursachen haben kann. Der erste mögliche Grund betrifft den $\mathrm{Zu}-$ sammenhang von Lohnpolitik und Zentralbankunabhängigkeit. In ihrem berühmten Aufsatz zeigen Calmfors und Driffill, dass sowohl stark zentralisierte als auch weit gehend dezentralisierte Lohnverhandlungssysteme eine moderate Lohnentwicklung gewährleisten, während intermediäre Systeme eine besonders schlechte makroökonomische Performanz aufweisen. ${ }^{35}$ Aus der Vergemeinschaftung der Geldpolitik ergibt sich aber eine relative Dezentralisierung der Lohnpolitik, die das Lohnverhandlungssystem der Euro-Zone in den ungünstigen intermediären Bereich rückt. In Deutschland führten die Lohnführerschaft der IG Metall und die glaubhafte Androhung der Bundesbank, inflationäre Lohnabschlüsse mit einer restriktiven Geldpolitik zu vergelten, zu einer impliziten Koordinierung von Geld- und Lohnpolitik. In der Währungsunion ist ein solches Zusammenspiel der Wirtschaftsakteure nicht gewährleistet. ${ }^{36}$ Mit der Dezentralisierung der Lohnpolitik fehlt der EZB der Adressat für die Geldpolitik, und es entsteht ein Trittbrettfahrerproblem zwischen den Gewerkschaften. Wenn der eigene Beitrag zur Gesamtinflation gering und gezielte Reaktion der EZB nicht

34 Issing, O.: Geldpolitik für den Euroraum, in: Perspektiven der Wirtschaftspolitik, 5/4 (2004), 381-404, hier 399.

35 Calmfors, L./Driffill, J.: Bargaining Structure, Corporatism and Macroeconomic Performance, in: Economic Policy, 3/6 (1988), 14-61.

36 Hall, P. A./Franzese, R. J.: Mixed Signals: Central Bank Independence, Coordinated Wage Bargaining, and European Monetary Union, in: International Organization, 52/3 (1998), 505-535; Iversen, T.: Wage Bargaining, Central Bank Independence, and the Real Effects of Money, in: International Organization, 52/3 (1998), 469-504. 
möglich ist, sinkt die Bereitschaft zur Lohnzurückhaltung. ${ }^{37}$ Eine Koordinierung der Lohnpolitik ist aus dieser Sicht zur Disziplinierung der Gewerkschaften wünschenswert.

Der zweite mögliche Grund für eine Koordinierung der Lohnpolitik liegt darin, dass in der Währungsunion die Wechselkurse als Anpassungsinstrument bei Zahlungsbilanzschwierigkeiten nicht mehr zur Verfügung stehen. Befindet sich ein Land dauerhaft in der Stagnation, kann die Wettbewerbsfähigkeit nicht über Abwertungen verbessert werden. Stattdessen muss diese Anpassung über die Preise erfolgen. Die Löhne dienen hierbei als funktionales Äquivalent einer Abwertung. Die Schwierigkeit, vor der die Lohnpolitik in diesem Szenario steht, liegt nicht in unverantwortlichen Lohnerhöhungen, sondern vielmehr in einem anhaltenden Unterbietungswettbewerb. Für Deutschland erscheint eine Steigerung der Wettbewerbsfähigkeit durch Lohnzurückhaltung besonders schwierig, weil sich viele Nachbarländer an der deutschen Lohnpolitik orientieren und versuchen, diese zu unterschreiten. ${ }^{38}$ Die Sozialen Pakte des letzten Jahrzehnts haben zudem alle Bemühungen der Gewerkschaften um eine einheitliche Lohnformel unterlaufen. Häufig willigen sie in nationale Wettbewerbskoalitionen ein, die einer europäisch abgestimmten Lohnpolitik widersprechen.

Die beiden genannten Punkte betreffen die horizontale Koordinierung der Lohnpolitik. Darüber hinaus gibt es aber auch daran anschließende vertikale Abstimmungserfordernisse. Der Wettbewerbskorporatismus birgt die Gefahr einer Deflation. Würden sich die deutschen Gewerkschaften auf Lohnkürzungen einlassen, folgten vermutlich viele der angrenzenden Länder. In einem solchen Fall sind der Zentralbank die Hände gebunden, weil eine Deflation weniger wirksam als die Inflation bekämpfen kann. Die EZB geht davon aus, dass ihr 2003 verändertes Inflationsziel („unter, aber nahe zwei Prozent“) Sicherheit dagegen bietet, ${ }^{39}$ obwohl dies ein im internationalen Vergleich niedriger Wert ist. Selbst wenn man dem Maastrichter assignment folgt, müsste eine koordinierte Lohnpolitik sowohl die Wettbewerbsposition einzelner Länder als auch die Inflationsentwicklung des

37 Hancké, B./Soskice, D.: Wage-Setting and Inflation Targets in EMU, in: Oxford Review of Economic Policy, 19/1 (2003), 149-160.

38 Milton Friedman verweist auf die Gefahr der politischen Rigidität von Löhnen und Preisen: „Adjusting to a change in the equilibrium real exchange rate via adjusting nominal prices and wages is far more difficult than adjusting via a change in the nominal exchange rate. [...] Germany's present difficulties are a foretaste of the difficulties that are likely to arise in the European Monetary Union as asymmetric shocks accumulate and one country after another finds that its prices and wages are not consistent with equilibrium in its foreign payments and receipts, even though they may have been when they entered the euro system" (Friedman, M.: Comment on Gaspar and Issing, in: Australian Economic Papers, 41/4 (2002), 366-368, hier 367).

39 ECB: The Outcome of the ECB's Evaluation, a. a. O., 79. 
gesamten Euro-Raums im Auge behalten. Erst sie würde das wirkungsvolle Zusammenspiel von Geld- und Lohnpolitik ermöglichen, das im Nationalstaat möglich war. Die Calmfors-Driffill-Analyse unterstreicht die positiven Effekte lohnpolitischer Koordinierung. Die Grundzüge der Wirtschaftspolitik und die Europäische Beschäftigungsstrategie fordern aber regelmäßig das Gegenteil. ${ }^{40} \mathrm{Im}$ Ergebnis verharrt die Euro-Zone im makroökonomisch ungünstigen Zwischenbereich.

Die Literatur, die sich mit der Frage nach dem Zusammenhang von koordinierter Lohnpolitik, Geldpolitik und makroökonomischer Performanz beschäftigt, begreift eine unabhängige Zentralbank nicht als per se überlegenes Modell, sondern verweist auf das Zusammenspiel verschiedener Politiken. ${ }^{41}$ Eine wirkungsvolle ex post-Koordinierung ist darauf angewiesen, dass die geldpolitischen Signale der EZB auf strategiefähige korporative Akteure stoßen. In der Euro-Zone sind die Adressaten jedoch fragmentiert. Während die EZB somit in der Lage ist, eine niedrige Inflationsrate durchzusetzen, muss dies nicht $\mathrm{zu}$ einem positiven Zusammenspiel der wirtschaftspolitischen Akteure führen. Das deutsche Modell der ex post-Koordinierung, auf dem die Währungsunion aufbaut, war institutionell voraussetzungsreich. Die Euro-Zone verfügt über kein analoges Institutionensystem, in das die Entscheidungen der EZB eingebettet sind.

\section{Das Korrespondenzproblem}

In diesem Abschnitt soll der Frage nachgegangen werden, ob die Aufteilung in verbindliche und weniger verbindliche Verfahren in der Währungsunion mit empirisch beobachtbaren negativen externen Effekten korrespondiert. Die Existenz möglicher spillovers begründet die fiskalpolitischen Regeln bezüglich der Defizite und Staatsschulden. So argumentiert die Europäische Kommission:

„[T] he rationale for economic policy co-ordination rests on the following grounds. Coordination is needed to take account of direct cross-border spillover of national policies on neighbouring countries. In addition, euro-area participants can also be affected indirectly by national policy actions. The average inflation rate and the exchange rate have become common goods. Thus, a national policy action that affects these variables can in turn impact the ECB policy decision on interest rates or the ECOFIN Council's judgment on exchanges rates. Moreover, co-ordination should help countering tempta-

Niechoj, T.: Attraktiv für Gewerkschaften? Keynesianische Makrokoordinierung auf EU-Ebene, in: Hein, E. u. a. (Hg.), a. a. O., 93-95.

41 McNamara, K.: Rational Fictions: Central Bank Independence and the Social Logic of Delegation, in: West European Politics, 25/1 (2002), 47-76. 
tion to resort to free-rider behaviour on the part of the Member States. Finally, besides economic arguments, coordination can also play a useful role from a political-economy viewpoint by helping to implement unpopular but necessary policy actions at national level.“42

Für die ersten Jahre der Währungsunion lässt sich allerdings kein Zusammenhang zwischen Defiziten und Inflationsraten feststellen (siehe Abbildung 2). Dieses Ergebnis kann nicht überraschen, weil es eine Vielzahl von Faktoren gibt, die auf die Inflation einwirken. Vor diesem Hintergrund erstaunt vielmehr, dass den Haushaltsdefiziten eine Sonderstellung hinsichtlich möglicher Sanktionen im EG-Vertrag zukommt und dass sowohl Kommission als auch EZB in ihren politischen Empfehlungen fast ausschließlich auf die Bedeutung der Defizite für die Inflation hinweisen.

Abbildung 2: Haushaltsüberschüsse und Inflationsraten, Euro-Länder, 1999-2004

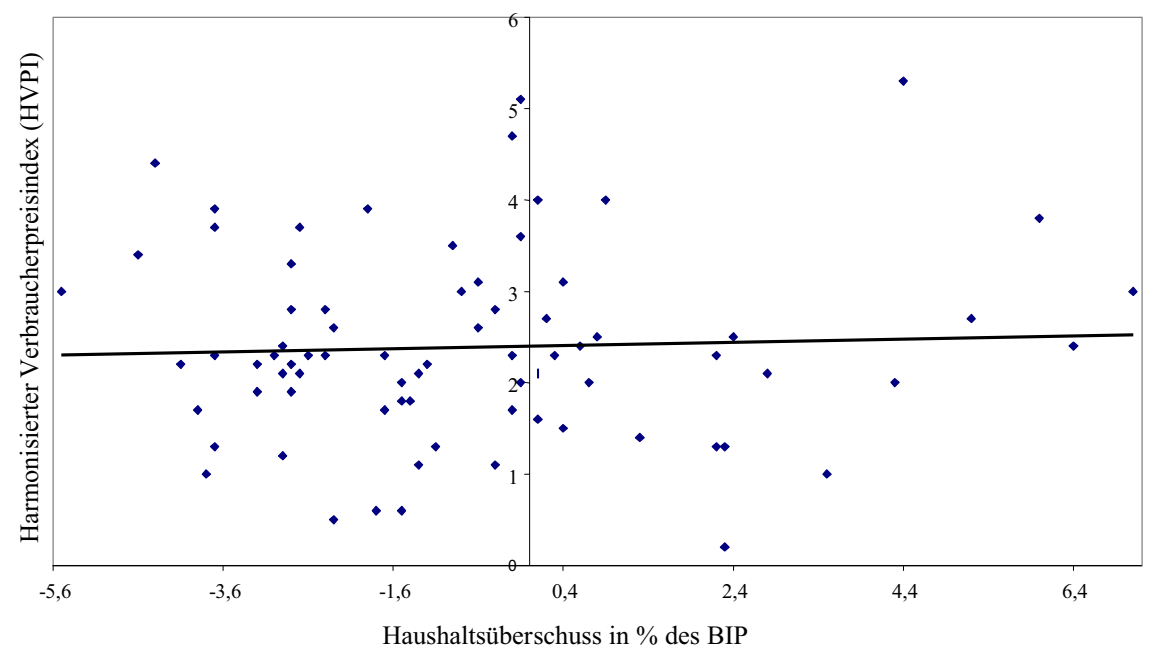

Quelle: Eurostat.

Die bestehenden Defizite werden nicht primär durch eine expansive Ausgabenpolitik erklärt, sondern durch die Ausgangslage zu Beginn der Währungsunion (siehe oben) sowie die anhaltende Wachstumsschwäche und das Wirken der automatischen Stabilisatoren (siehe Abbildung 3). Deshalb schlägt etwa der Internationale Währungsfonds für die Euro-Zone eine schrittweise Reduzierung der strukturellen Defizite vor, auch wenn dies aufgrund der konjunkturellen Entwicklung bedeutet, dass weitere Länder den SWP brechen. ${ }^{43}$

42 European Commission, Directorate-General for Economic and Financial Affairs, a. a. O., 3 f.

43 IMF: Euro Area Policies, in: IMF Country Report Nr. 297 (2003), Washington. 
Abbildung 3: Wachstum und Haushaltsdefizit, Euro-Zone, 1999-2004

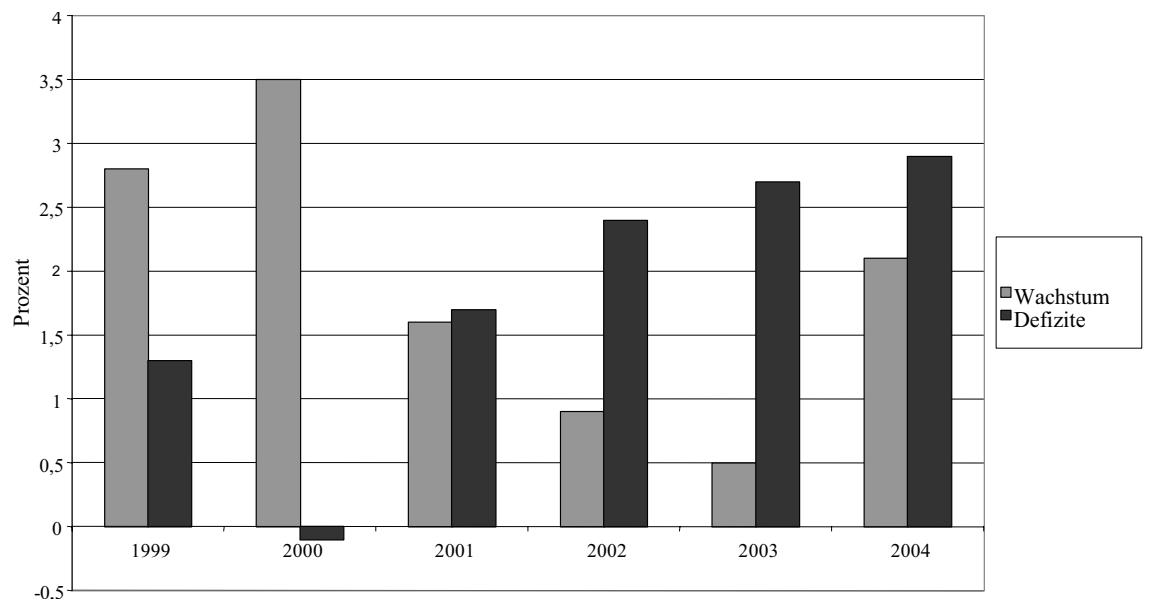

Quelle: Eurostat.

Eine unverantwortliche Haushaltspolitik der Euro-Staaten gefährdet nach Ansicht von EZB und Europäischer Kommission den Außenwert der Währung. Auch hier unterstützt die bisher vorliegende Evidenz das Argument nicht. Seit 2001 steigen nicht nur die Defizite kontinuierlich an, sondern auch der effektive Euro-Wechselkurs (Abbildung 4). Natürlich beweist dieser Anstieg nicht, dass kein Zusammenhang zwischen Defiziten und Außenwert besteht, weil der Wechselkurs auch durch die Politik der Drittstaaten bestimmt wird. Aber die Entwicklung der vergangenen Jahre stellt zumindest die einfache Formel infrage, dass Defizite Folge einer unsoliden Haushaltspolitik sind, die die innere und äußere Stabilität des Euros gefährdet.

Auch wenn die betrachtete Zeitspanne für robuste Schlussfolgerungen zu kurz ist, weckt die wirtschaftliche Entwicklung der letzten Jahre Zweifel an der von EZB und Kommission genannten Begründung für die Regeln der Währungsunion. Diese Kritikpunkte lassen sich wie folgt zusammenfassen:

- Die Übertragung der Geldpolitik auf die Europäische Zentralbank stellt ein wirksames Mittel zur Vermeidung von Inflation dar. Sie alleine garantiert aber keine wirkungsvolle ex post-Koordinierung, weil die Dezentralisierung der Fiskal-, Arbeitsmarkt- und Lohnpolitik vertikale und horizontale Koordinierungsprobleme aufwirft.

- Die Regeln des SWP bieten kein Instrumentarium für den Übergang zu mittelfristig ausgeglichenen Haushalten, sondern wirken im ungünstigsten Fall diesem Ziel entgegen. Ohne eine wirksame ex ante-Abstimmung müssen die nationalen Regierungen die Kosten für die Haushaltskonsolidierung in vollem 
Umfang selbst tragen. Eine autonome Zentralbank kann den Abbau der Defizite erschweren.

- Strukturreformen müssen zwischen den Mitgliedstaaten abgestimmt werden, damit die EZB darauf reagieren kann. Bei diffusen Signalen der Mitgliedstaaten ist dies nicht möglich. Kommission und EZB heben regelmäßig die Bedeutung derartiger Reformen hervor, aber die Grundzüge der Wirtschaftspolitik enthalten keine Sanktionsmöglichkeit und können keine Gewähr für ein konzertiertes Vorgehen bieten.

- Die unkoordinierte Lohnpolitik in der Euro-Zone kann eine Reihe negativer Folgen haben. Dennoch gibt es in diesem Bereich keine vertragliche Grundlage für eine engere Zusammenarbeit. Die arbeitsmarktpolitischen Empfehlungen der Grundzüge der Wirtschaftspolitik sowie der Beschäftigungsstrategie fordern eine weitere Dezentralisierung.

Abbildung 4: Wechselkurs des Euro in \% des Ausgangswerts, 1999-2004

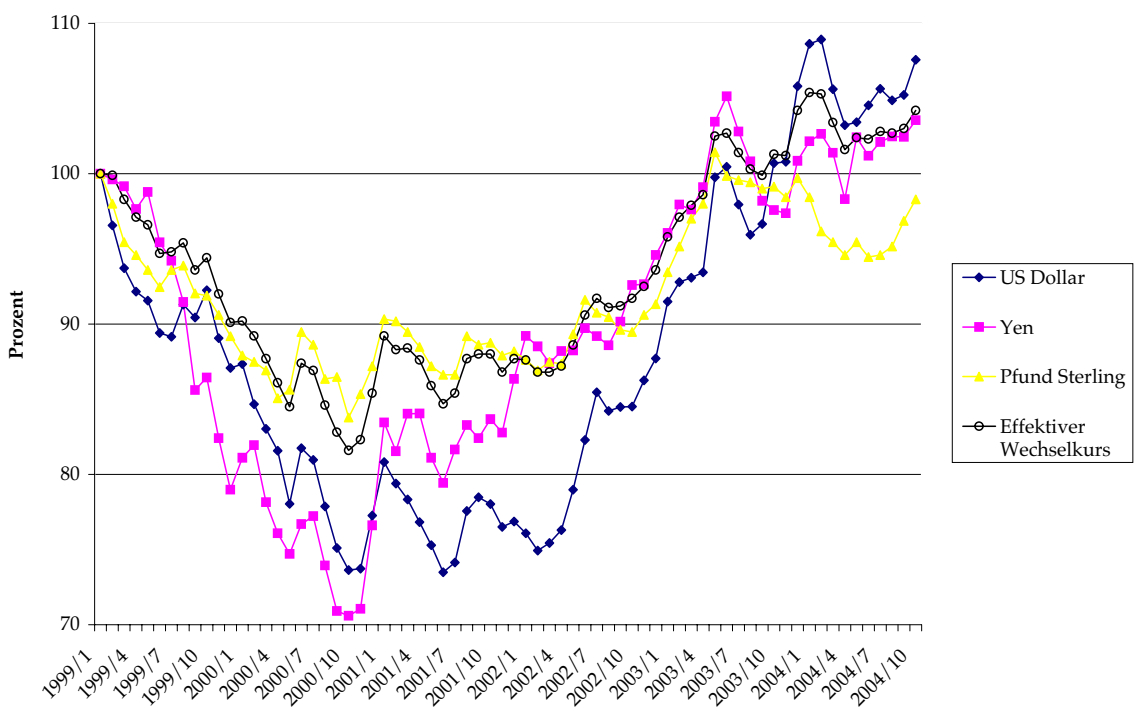

Quelle: ECB Monatsberichte, verschiedene Ausgaben.

Anmerkung: Den realen effektiven Wechselkurs ermittelt die EZB auf Basis der gewichteten Durchschnitte der bilateralen Wechselkurse des Euro gegenüber den Handelspartnern der Euro-Länder. Die Ländergruppe umfasst die 13 nicht dem Euro-Währungsgebiet angehörenden EU-Staaten sowie Australien, China, Hongkong, Japan, Kanada, Norwegen, die Schweiz, Singapur, Südkorea und die USA.

Zusammengenommen widerspricht die Analyse des Maastrichter assignment der Annahme, dass die unterschiedlichen Koordinierungsinstrumente vorwiegend aufgrund wirtschaftlicher Überlegungen gewählt wurden. Die EZB-Veröffentlichungen verweisen stattdessen auf ein grundlegendes Misstrauen gegenüber 
politischen Entscheidungen. Nationalen Regierungen und Verwaltungen wird ein säkularer Trend zu höheren Ausgaben und damit zu höherer Inflation unterstellt, der nur durch externe Restriktionen eingedämmt werden kann. Empirische Untersuchungen zeigen dagegen, dass Inflation treibende Politiken mit der Abkehr vom Keynesianismus seit den 1970er Jahren zurückgegangen sind, und die Bedeutung unabhängiger Zentralbanken zur Gewährleistung dieses Ziels im Zeitverlauf abgenommen hat. ${ }^{44}$ Das Maastrichter assignment soll die Wirtschaftsakteure zu regelgebundenem Verhalten anhalten und die Politik zurückdrängen. Allerdings wird die Maxime der Regelbindung ungleich auf die Wirtschaftsakteure angewandt. Die EZB verfügt in der Geldpolitik über erhebliche Entscheidungsfreiheit. Begründet wird diese Ausnahme mit dem nicht-opportunistischen, nicht-politischen Verhalten der Zentralbank und mit der Neutralität des Gelds. In der Konstitutionalisierung von regelgebundener Haushaltspolitik und Zentralbankunabhängigkeit greifen die Verhaltensannahmen der ökonomischen Theorie der Politik. Budgetmaximierung ist aus dieser Perspektive ein unausweichliches Motiv politischer Entscheidungsträger ${ }^{45}$ Unerklärt bleibt allerdings, weshalb die Adressaten der Regeln als deren Autoren zur Einschränkung ihrer Handlungsfähigkeit bereit sind - denn warum sollten sie sich ihre Handlungsfreiheit gegen den eigenen Willen durch europäische Vorgaben einschränken lassen?

Die Währungsunion ist das Ergebnis politischer Aushandlungsprozesse. Viele der zitierten Studien rationalisieren die bestehenden Institutionen, erklären können sie ihr Entstehen damit jedoch nicht. Zudem ist in der ökonomischen Logik ein weiteres Zurückdrängen politischer zugunsten technokratischer Entscheidungen angelegt, das jedoch mit erheblichen Legitimitätsproblemen konfrontiert ist. Auch wenn nicht klar ist, ob zukünftig weitere Bereiche der Wirtschaftspolitik wie zuvor die Geldpolitik - dem politischen Zugriff entzogen werden, erscheint es dennoch geboten, dass die Politikwissenschaft ökonomische Argumente nicht unhinterfragt akzeptiert, um sich weiter ungestört an der deliberativen Qualität peripherer Verfahren erfreuen zu können. Im abschließenden Abschnitt wird eine politikwissenschaftliche Erklärung der Wahl harter oder weicher Verfahren skizziert.

44 Kaltenthaler, K. C./Anderson, C. J.: The Changing Political Economy of Inflation, in: Journal of Public Policy, 20/2 (2000), 109-131.

45 Niskanen, W. A.: Bureaucracy and Representative Government, Chicago/New York, 1971. 


\section{Ansätze zu einer politikwissenschaftlichen Erklärung: anhaltende Integrationsmüdigkeit}

Die Mitgliedstaaten der EU haben sich in einem Jahrzehnt auf die Europäische Währungsunion geeinigt (1991), den Stabilitäts- und Wachstumspakt verabschiedet (1996/97), einen Beschäftigungstitel in den EG-Vertrag eingefügt (1997) sowie die Offene Methode der Koordinierung als neues Politikinstrument eingeführt (2000). Während die Geldpolitik vergemeinschaftet und ein (formal) hartes Koordinierungsverfahren für die Haushaltspolitik festgelegt wurde, beschränken sich Europäische Beschäftigungsstrategie und OMK auf eine unverbindliche, sanktionslose Koordinierung, die der multilateralen Überwachung von OECD und Internationalen Währungsfonds gleicht. Diese Unterschiede lassen sich nicht ausschließlich, so das Argument des vorherigen Abschnitts, mit der Bedeutung erklären, die diese Politikfelder für den policy mix der Euro-Zone haben. Auch in der Wirtschafts-, Arbeitsmarkt- und Lohnpolitik gibt es Argumente für eine stärkere Koordinierung, die jedoch politisch nicht durchsetzbar waren. Die selektive Vergemeinschaftung in der europäischen Wirtschafts- und Arbeitsmarktpolitik folgt nicht zuvorderst funktionalen Erfordernissen. Die Frage, die wir an die politische Ökonomie der Währungsunion richten müssen, ist daher jene der klassischen Integrationstheorie: Unter welchen Umständen sind Regierungen zur Abgabe von Souveränität bereit?

Der erste Faktor, der Beachtung verdient, ist der Wandel des dominanten wirtschaftspolitischen Paradigmas seit den 1970er Jahren. Die europäischen Staaten bemühten sich seit dieser Zeit um die Stabilisierung der Wechselkurse mit dem Ziel einer späteren Währungsunion. Diese Bemühungen scheiterten daran, dass Hart- und Weichwährungsländer, vor allem Deutschland und Frankreich, einander widersprechende Vorstellung von der Umsetzung der Währungsunion hatten. ${ }^{46}$ Erst in den $1980 e r$ Jahren setzte sich das Primat der Preisstabilität europaweit durch. Die institutionelle Voraussetzung für Geldwertstabilität war, so schien das deutsche Beispiel zu zeigen, eine unabhängige Zentralbank. Mit der neuen Austeritätspolitik und dem Abschied vom Vollbeschäftigungsprimat wurde eine institutionell schlanke Version der Währungsunion möglich, zumal steuernde Eingriffe in die Wirtschaft ohnehin wenig Erfolg versprechend schienen. Der externe Anker zuerst des Europäischen Währungssystems und dann der Währungsunion entlastete die Regierungen von gesellschaftlichen Ansprüchen an ihre Leistungsfähigkeit. ${ }^{47}$ Ein entscheidendes Resultat dieser Neuorientierung war, dass

46 Kruse, D. C.: Monetary Integration in Western Europe: EMU, EMS and Beyond, London u. a., 1980.

47 Siehe Notermans, T.: The Abdication from National Policy Autonomy: Why the Macroeconomic Policy Regime Has Become so Unfavorable to Labor, in: Politics \& Society, 21/2 (1993), 133-167. 
für die meisten Staaten die Währungsunion ohne nennenswerte Souveränitätsverluste möglich wurde. Sie hatten schon zuvor ihre geldpolitische Autonomie zugunsten fester Wechselkurse abgegeben. Frühere Vorschläge für die Währungsunion hatten dagegen weit gehende Kompetenztransfers auf die europäische Ebene gefordert und waren damit auf den Widerstand nationaler Regierungen gestoßen. ${ }^{48}$ Erst das Delors-Komitee konnte sich eine Währungsunion ohne Wirtschaftsregierung vorstellen, womit den Regierungen die Zustimmung erheblich erleichtert wurde.

Für die Ausgestaltung der Währungsunion war neben der Konvergenz der Wirtschaftspolitik (vor allem: Disinflation) die besondere Position Deutschlands entscheidend. Da eine gemeinsame europäische Währung vor allem für Deutschland einen materiellen Gestaltungsverlust bedeutete, sie ohne das wirtschaftlich stärkste Land jedoch undenkbar blieb, konnte die deutsche Bundesregierung im Zusammenspiel mit der Bundesbank die Gestaltung der Währungsunion im Kern bestimmen. Der Erklärungsfaktor für die Wahl harter währungspolitischer Institutionen lag in der Fähigkeit Deutschlands, seine Präferenz durchzusetzen, obwohl dieser selektive Institutionentransfer umstritten war. ${ }^{49}$ Mit dem Stabilitätsund Wachstumspakt wiederholte sich das Muster der Entscheidung für die Währungsunion. Auch er wurde vom monetaristischen Konsens getragen, wie eine stabile Währung am besten gesichert werden könne. Dieser Konsens reichte jedoch nicht aus, die Ausgestaltung des SWP zu bestimmen. Hinzu kam die dominierende Verhandlungsposition Deutschlands, das - innenpolitisch motiviert - auf strikten Haushaltsregeln beharrte, und hierbei Unterstützung durch die Bundesbank fand. ${ }^{50}$

Getragen wurden die Maastrichter Verhandlungen zudem von einer ungewöhnlich breiten Mehrheit von wirtschaftsfreundlichen Regierungen. Zu dieser Zeit waren zehn von zwölf Mitgliedstaaten von Mitte-Rechts-Parteien regiert, häufig von christdemokratisch-liberalen Koalitionen. Vor allem die Christdemokraten arbeiteten zusammen und trafen sich regelmäßig, um ihre Vorstellung effektiv durchzusetzen. Die drei federführenden Ratspräsidentschaften bis zur Verabschiedung des Maastrichter Vertrags hielten Italien, Luxemburg und die Niederlande. Alle drei hatten zu dieser Zeit christdemokratische Premierminister. Die enge Verbindung und ideologische Übereinstimmung entscheidender Akteure erleichterte die Einigung auf die Währungsunion. ${ }^{51}$ Die verhandelnden

48 Ludlow, P.: The Making of the European Monetary System, London u. a., 1982, 4.

49 Dyson, K./Featherstone, K.: The Road to Maastricht, Oxford, 1999, 794.

50 Heipertz, M./Verdun, A.: The Dog That Would Never Bite? What Can We Learn From the Origins of the Stability and Growth Pact, in: Journal of European Public Policy, 11/5 (2004), 765-780.

51 Ausführlicher zur Bedeutung von Parteien als Erklärungsfaktor für die europäische Integration: Manow, 
Mitte-Rechts-Regierungen wollten Institutionen zur dauerhaften Disziplinierung ihrer Nachfolger schaffen. Nach Maastricht verloren die Christdemokraten die Mehrheit, und immer mehr Länder wurden sozialdemokratisch regiert. In Amsterdam und Lissabon gab es eine klare Mehrheit links von der Mitte. Die Wahlerfolge der Sozialdemokratie waren die Voraussetzung für die Aufnahme eines Beschäftigungstitels in den EG-Vertrag, sie brachten aber eine ideologisch heterogene neue Sozialdemokratie an die Regierung. In der Substanz der Beschäftigungsstrategie, über mögliche Kompetenztransfers sowie die Verbindlichkeit des Verfahrens gab es zwischen den Regierungen keine Übereinstimmung. Die französischen Sozialisten und New Labour in Großbritannien verfolgten auf nationaler Ebene unterschiedliche Arbeitsmarktpolitiken und konnten sich nicht auf einen gemeinsamen europäischen Ansatz einigen. Das weiche Überwachungsverfahren des Beschäftigungstitels wurde gewählt, um trotz dieser Differenzen das arbeitsmarktpolitische Profil der EU zu stärken. ${ }^{52}$ Besonders nordeuropäische Sozialdemokraten lehnten die Abgabe zentraler Kompetenzen an Brüssel ab.

Aus den bisher genannten Faktoren lässt sich eine politische Erklärung für die Wahl weicher und harter Koordinierungsverfahren ableiten. Die Geschichte der europäischen Integration und vor allem die Einigung auf die Währungsunion sind deshalb so bemerkenswert, weil sie vom sonst üblichen Muster zwischenstaatlicher Zusammenarbeit abweichen. Doch der weit gehende Souveränitätstransfer der Währungsunion ist selbst im EU-Kontext ungewöhnlich. Eine integrationswillige und liberale parteipolitische Mehrheit in den Mitgliedstaaten, der Zusammenbruch des Ostblocks mit der Möglichkeit der deutschen Wiedervereinigung sowie die Sonderstellung der Geldpolitik ermöglichten in Maastricht die Zustimmung zu einer Währungsunion, bei der die Geldpolitik zentralisiert wurde, aber ansonsten der Kompetenztransfer auf die europäische Ebene gering blieb. In anderen Feldern der Wirtschaftspolitik waren die Regierungen der Mitgliedstaaten nicht zum Souveränitätsverzicht bereit. Mit der Beschäftigungsstrategie und der OMK wählten sie stattdessen Formen der Kooperation, die ohne Souveränitätstransfers auskamen. Weiche Koordinierung baut auf der Prämisse nationaler Zuständigkeit auf. Die Wahl weicher Koordinierungsverfahren in der Wirtschaftspolitik wird nicht durch die ökonomische Logik bestimmt, sondern durch den Unwillen der Mitgliedstaaten zur Abgabe weiterer Souveränität. ${ }^{53}$

P./Schäfer, A./Zorn, H.: European Social Policy and Europe's Party-Political Center of Gravity, 1957-2003, MPIfG Discussion Paper 04/6, Köln, 2004.

52 Vgl. aber Merkel, W.: Die Dritten Wege der Sozialdemokratie ins 21. Jahrhundert, in: Berliner Journal für Soziologie, 10/1 (2000), 99-124, hier 117-120.

53 Hagen, J. v./Mundschenk, S.: The Functioning of Economic Policy Coordination, ZEI Working Paper Nr. 8 , Bonn, 2001, $23 \mathrm{f}$. 
Für die Phase nach Maastricht lässt sich von einer Integrationsmüdigkeit der Regierungen sprechen. Die Währungsunion war ein so deutliches Zeichen für die Abnahme nationaler Gestaltungsfähigkeit, dass die Regierungen sich in den anschließenden Regierungskonferenzen weiterem Souveränitätstransfer widersetzten. Die entscheidende Neuerung nach Maastricht war die Einführung des Beschäftigungstitels in den Amsterdamer Vertrag. Die dort festgelegte Koordinierungsform besteht darin, gemeinsame Ziele zu formulieren, ohne die Regierungen rechtlich auf sie verpflichten zu können. Die offene Methode der Koordinierung vermindert den Einfluss supranationaler Akteure. Europäisches Parlament und Europäischer Gerichtshof verfügen über keine Befugnisse, und auch die Kommission kann keine Entscheidungen gegen den Willen der Mitgliedstaaten treffen. Missachtet eine Regierung die europäischen Politikempfehlungen, bleibt nur die Möglichkeit, sie öffentlich bloßzustellen (naming and shaming) doch im Gegensatz zur Pisa-Studie der OECD werden diese Versuche in der EU von der Öffentlichkeit kaum wahrgenommen. Die Vertragsrevision von Nizza und der Verfassungskonvent hatten vor allem die Straffung bestehender Entscheidungsverfahren zum Ziel. Sie beinhalteten jedoch wenige Kompetenzverlagerungen auf die europäische Ebene. Gleichzeitig fand eine erhebliche Ausweitung der Anwendungsfelder weicher Koordinierung statt. Vor dem Hintergrund der hier vorgeschlagenen Perspektive auf die Wahl unterschiedlicher Koordinierungsinstrumente erscheinen diese Entwicklungen als Ausdruck einer stärkeren Zurückhaltung der Regierungen, sich in weiteren Politikfeldern die Hände zu binden. Die veränderten Regeln des Stabilitäts- und Wachstumspaktes zeigen, dass Regierungen in Defizitländern nicht bereit sind, harte europäische Vorgaben in wirtschaftlich schwierigen Zeiten hinzunehmen. Das Aufweichen der Vereinbarung erhöht erneut den diskretionären Spielraum nationaler Politik - auch hier dominiert nicht die ökonomische, sondern eine politische Logik.

Die vorangegangenen Ausführungen können nicht entscheiden, ob eine stärkere Koordinierung der Wirtschaftspolitik zu besseren Ergebnissen führen würde, da dies eigene Probleme - vor allem zeitinkonsistenten Verhaltens - aufwürfe. Aber sie wecken Zweifel an der überlegenen Leistungsfähigkeit des Maastrichter assignment und damit an der Begründung für die bestehende Aufteilung in weiche und harte Koordinierungsverfahren. Die Abschottung der Geldpolitik wird häufig durch ihre gesamtwirtschaftlich positiven Effekte gerechtfertigt. Wenn die institutionellen Voraussetzungen dafür in der Währungsunion jedoch unvollständig gegeben sind, verliert sie einen wichtigen Teil ihrer output-Legitimität. Schließlich wurde die Währungsunion mit dem Versprechen auf ein dauerhaft höheres Wachstum begründet. Zehn Jahre später ist diese Zielvorstellung auf die OMK übertragen worden, die nun Europa zum wettbewerbsfähigsten Wirt- 
schaftsraum der Welt machen soll. Da beide Versprechen noch auf ihre Einlösung warten, sollte sich die Politikwissenschaft nicht durch die einseitige Konzentration auf „weiche“ Verfahren die Möglichkeit nehmen, die Institutionen der Währungsunion aus wirtschaftspolitischer und demokratietheoretischer Sicht zu kritisieren. 